\title{
Os significados acerca da Estratégia Saúde da Família para uma comunidade
}

RESUMO | Objetivos: identificar os significados acerca da Estratégia Saúde da Família para uma comunidade. Método: estudo qualitativo, exploratório, descritivo e transversal, amostragem não probabilística e intencional, realizado com 20 participantes, com a utilização de dois instrumentos. Os dados das entrevistas foram avaliados considerando a abordagem do Discurso do Sujeito Coletivo pautados na Teoria das Representações Sociais. Quanto aos dados do questionário foram tratados de forma percentual. O estudo foi aprovado pelo Comitê de Ética em Pesquisa. Resultados: 75\% eram do gênero feminino, faixa etária de 61 a 70 anos, 30\% possuem ensino médio completo e 6 meses a 10 anos em que residem próximo a ESF. Emergiram duas ideias centrais "muito bom" e "nenhum". Conclusão: a maioria dos entrevistados atribuiu significados positivos diante da inserção da unidade em sua área de moradia. Porém, apesar da facilidade de acesso, ressaltam a necessidade de melhorias nos serviços.

Palavras-chaves: Estratégia Saúde da Família; Atenção Primária à Saúde; Pesquisa Qualitativa.

ABSTRACT | Objectives: to identify the meanings about the Family Health Strategy for a community. Method: the study is a qualitative, exploratory, cross-sectional, non probabilistic and intentional sampling, composed with 20 participants using two instruments. The data from the interviews were evaluated considering the collective subject discourse approach based on the Theory of Social Representations. Regarding the data from the questionnaire, they were treated in a percentage way. The study was approved by the Research Ethics Committee. Results: 75\% were female, aged 61 to 70 years, 30\% had completed high school and 6 months to 10 years in which they live near the ESF. Two central ideas emerged "very good" and "none". Conclusion: the most of the interviewees attributed positive meanings to the insertion of the unit in their housing area. However, despite the ease of access, they still emphasize the need for improvements in services.

Keywords: Family Health Strategy; Primary Health Care; Qualitative Research.

RESUMEN | Objetivos: Identificar los significados sobre la Estrategia de Salud Familiar para una comunidad. Método: Estudio de enfoque cualitativo, exploratorio, descriptivo y transversal, muestreo no probabilístico e intencional, con un total de 20 participantes, con el uso de dos instrumentos. Los datos de las entrevistas fueron evaluados considerando el enfoque del discurso de sujeto colectivo basado en la Teoría de las Representaciones Sociales. En cuanto a los datos del cuestionario, fueron tratados de manera porcentual. El estudio fue aprobado por el Comité de ética de la investigación. Resultados: el 75\% eran mujeres, de 61 a 70 años, el 30\% habían completado la escuela secundaria y 6 meses a 10 años en los que viven cerca del FSE. Dos ideas centrales surgieron "muy buenas" y "ninguna". Conclusión: La mayoría de los entrevistados atribuyeron significados positivos a la inserción de la unidad en su área de vivienda. Sin embargo, a pesar de la facilidad de acceso, siguen insistiendo en la necesidad de mejorar los servicios.

Palabras claves: Estrategia de Salud Familiar; Atención Primaria de Salud; Investigación Cualitativa.

\section{Fábio Luiz Piedade}

Graduando em Enfermagem na Faculdade

Wenceslau Braz.

ORCID: 0000-0001-5347-8225

\section{Pâmela Alves dos Santos}

Graduanda em Enfermagem na Faculdade Wenceslau Braz.

ORCID: 0000-0003-1223-056X

\section{Jerusa Gomes Vasconcellos Haddad}

Enfermeira. Mestra em Ciências pela Escola de Enfermagem da Universidade de São Paulo. Professora e Coordenadora Acadêmica do Curso de Enfermagem da Faculdade Wenceslau Braz.

ORCID: 0000-0001-6170-064X

Recebido em: 14/09/2020

Aprovado em: 10/12/2020

INTRODUÇÃO

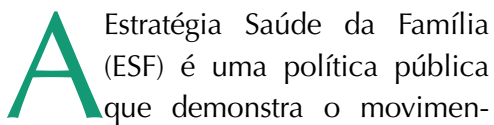

to de ampliação da rede assistencial no Sistema Único de Saúde (SUS), por meio do fortalecimento da proposta da Atenção Primária à Saúde (APS) ${ }^{1}$. Durante essa ampliação dos serviços de APS, os Centros de Saúde da Família (CSF) vêm se moldando com espaços novos para o desenvolvimento de diferentes práticas profissionais.

A ESF se caracteriza por uma atenção territorializada, desenvolvida por equipes multiprofissionais responsáveis pelo planejamento de ações de acordo com as necessidades locais de uma comunidade ${ }^{2}$.

A unidade possui conceitos amplos quando se trata de saúde e compreensão dos determinantes do processo saúde-doença. Propondo uma forma de articulação entre os saberes técnicos e provenientes da população e a mobilização de recursos institucionais e comunitários para que haja o enfrentamento dos problemas de saúde.

Como novo modelo ela se responsabiliza integralmente pelas necessidades de saúde de um determinado local, da mesma forma com que dita uma reorganização do modelo assistencial de saúde brasileiro, tendo como base princípios de universalidade, equidade e integralidade.

A proposta trazida pela ESF produz diferentes resultados assistenciais por incorporar novas ações de saúde no campo de atuação individual e coletivo, incluindo-se a promoção, proteção, prevenção, diagnóstico, tratamento, reabilitação, re- 
dução de danos e a manutenção da saúde de seus usuários ${ }^{3}$.

No entanto, a dicotomia e oposição entre a assistência e a promoção da saúde, consiste ainda em um desafio para esse modelo assistencial. A compreensão de que a saúde possui múltiplos determinantes e condicionantes e que a melhora das condições de saúde das pessoas e coletividades passa por diversos fatores, que são cabíveis de serem abordados na ESF, remete a superação desse desafio ${ }^{4}$.

O estudo é de grande relevância para os profissionais de saúde, pois os dados obtidos nesta pesquisa irão contribuir para sua atuação no sentido de nortear suas ações, contribuindo para a melhoria da assistência prestada. Onde toda a sociedade se beneficiará já que a melhoria da assistência prestada implica diretamente na melhoria da qualidade de vida da população.

Diante do exposto, esta pesquisa teve como objetivo identificar os significados acerca da Estratégia Saúde da Família para uma comunidade de um município do sul de Minas Gerais.

\section{MÉTODOS}

Trata-se de uma pesquisa qualitativa, exploratória, descritiva e transversal. A amostra foi intencional, composta por vinte moradores da área de abrangência da Estratégia Saúde da Família (ESF) de uma cidade do Sul de Minas Gerais, que preenchiam os seguintes critérios de inclusão: ser morador da área de abrangência da ESF há pelo menos seis meses, ser usuário da ESF; ter 18 anos ou mais, aceitar em participar do estudo assinando o Termo de Consentimento Livre e Esclarecido (TCLE).

A coleta de dados foi realizada com o auxílio de dois instrumentos: entrevista semiestruturada, contendo uma questão norteadora: "Se um amigo lhe perguntasse o que significa para você ESF, o que você responderia? E, um questionário para registro das características sociodemográficas dos participantes. Os dados foram coletados entre os meses de junho e julho de 2017.

As respostas derivadas das entrevistas foram interpretadas por meio da técnica do Discurso do Sujeito Coletivo (DSC). O DSC é um método de organização e tabulação dos dados qualitativos resultante de um discurso resumido, redigido na primeira pessoa do singular e organizado de acordo com os depoimentos semelhantes ${ }^{5}$. Foram aplicadas como figuras metodológicas: a expressão-chave (E-Ch); a ideia central (IC); e o Discurso do Sujeito Coletivo (DSC). A E-Ch revelam os depoimentos sobre o tema investigado, e a partir delas foram evidenciadas as IC, que descreveram o sentido de cada discurso analisado.

O DSC fundamenta-se na Teoria das Representações Sociais e se resume em analisar as ideias centrais, ancoragens e expressões-chave semelhantes dos discursos ${ }^{6}$.

As entrevistas foram gravadas e transcritas mantendo a fidedignidade das informações coletadas. Os dados sociodemográficos foram analisados e descritos de forma percentual.

Este estudo respeitou-se os preceitos éticos da Resolução 466/127 e obteve a aprovação do Comitê de Ética em Pesquisa da Faculdade Wenceslau Braz, sob o protocolo n. 2.358.117, CAAE: 78871317.3.0000.5099.

\section{RESULTADOS}

Fizeram parte do estudo 20 participantes com as seguintes características: $75 \%$ eram do gênero feminino, faixa etária de maior prevalência foi de 61 a 70 anos, frequência relativa de 35\%, 30\% possuem ensino médio completo, $25 \%$ possuem ensino fundamental completo e $10 \%$ possuem ensino superior. Em relação ao tempo em que moram nas proximidades da área da ESF com maior prevalência foi de 6 meses a 10 anos, frequência relativa de $30 \%$.

$\mathrm{Na}$ avaliação dos dados qualitativos evidenciaram as seguintes ideias centrais: "Muito bom"; "Nenhum". $1^{\mathrm{a}}$ IC - Muito bom

A primeira IC derivada dos discursos dos participantes afirmou que a proximidade da unidade com o local onde moram facilita o acesso da comunidade aos serviços de saúde. Dessa forma, evitando o congestionamento de outras unidades e auxiliando usuários que possuem doenças crônicas e necessitam de um controle diário, como descreve os DSCs a seguir.

"Muito bom mesmo, a melhor coisa que fizeram é o posto perto da minha casa [...]. Quando veio o posto pra cá melhorou bastante [...]. É uma coisa boa, porque facilita o acesso dos moradores [...]. [...] estou fazendo um controle de diabetes que eu descobri aqui. Então tudo isso é uma boa também. O pessoal depende muito daqui, ajuda principalmente as pessoas mais idosas. [...] No comecinho estava meio complicadinho, porque passava um tempinho para consulta, agora não, ai como é retorno, mostrar exames, eles marcam para gente mesmo [...]. Os problemas que a gente enfrenta aqui normalmente é a falta de vagas e se conseguir marcar é atendido, mas o atendimento é bom, e quando eles estão com alguma emergência a gente vem é feito uma triagem [...]. Para mim, que sou hipertensa e diabética, tenho facilidade [...]. [...] acho que isso é um direito de todos nós cidadãos. [...] a implantação foi boa, mais precisamos de melhoria, tanto como uma farmácia disponível, quanto aos médicos especialistas que todos postos possuem.

$2^{\mathrm{a}}$ IC - Nenhum

A segunda IC obtida a partir do significado acerca da Estratégia Saúde da Família para a comunidade foi "Nenhum". Esta ideia foi evidenciada pelo DSC:

Nenhum, já faz um ano eu nun- 
ca consegui consulta aqui. Agora eu vim aqui para conversar com a enfermeira chefe, porque eu vou transferir minhas crianças. Com esse negócio de você mora aqui tem que ser aqui, eu acho errado isso. Consulta mesmo para mim eu nunca consegui, faz um ano, toda vez que chego aqui, não tem vaga mais, começa marcar 1 hora, mas você chega aqui 10 horas já está lotado. Complicado, aqui é enrolado.

\section{DISCUSSÃO}

No presente estudo a IC "muito bom" é evidenciada pelos participantes onde afirmam que a ESF próximo à residência favorece o acesso aos serviços de saúde, evita a superlotação das outras unidades, facilita para aqueles que precisam de atendimento contínuo e auxílio para tratar as doenças crônicas.

A atenção primária é um elemento fundamental do sistema de saúde. Sendo consolidada por meio de seus índices e impactos que mostram melhorias na saúde da população de países que adotaram suas políticas. Dentro das evidências podem ser citados os melhores indicadores de saúde, melhor dinâmica do fluxo de usuários no sistema, melhoria no tratamento de doenças crônicas, avanço das práticas preventivas, aumento da satisfação de seus usuários e diminuição das dificuldades assim como iniquidades a respeito do acesso aos serviços. Além de elevar a eficiência do cuidado ${ }^{8}$.

A ESF tem atuado, desde sua implantação, na prevenção de doenças crônicas não transmissíveis, com ênfase na hipertensão, diabetes e câncer ginecológico. Tendo um importante papel no fortalecimento das ações de promoção a saúde no que se refere ao cuidado integral. A partir de sua implantação a ESF tem apontado vários avanços na assistência, especialmente quando nos referimos às ações de saúde ofertadas, ao acesso e uso dos serviços, queda das taxas de óbito por doenças infecto parasitárias e mortalidade infantil, juntamente com internações causadas por diarreia. As evidências indicam que este impacto positivo nos indicadores vem sendo sustentado ao longo do tempo ${ }^{9}$, podendo exercer um papel central na epidemia global de doenças cardiovasculares que vem crescendo ultimamente. Tanto na promoção da melhoria da saúde cardiovascular como no combate a patologias que afetam o sistema cardíaco ${ }^{10}$.

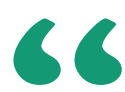

para mais perto de seus usuários, contanto com a implementação de agentes comunitários que vão até os moradores e certificam de que estes sejam cadastrados e regularizados no Sistema Básico de Saúde. O processo de trabalho visa e garante o maior acesso possível a população, além de fortalecer o vínculo entre seus usuários e profissionais. Possibilita, dessa forma, a continuidade e coordenação do cuidado, fazendo com que o usuário seja acompanhado ao longo do tempo pela equipe de saúde. Suas diretrizes estabelecem parâmetros que determinam o número máximo de famílias a serem abrangidas, evitando assim a superlotação das unidades e o desgaste dos profissionais atuantes nas áreas ${ }^{2}$.

Ainda referente primeira IC os participantes destacaram a importância da ESF para moradores idosos quem compõem a área de abrangência da unidade.

A ESF foi planejada com o objetivo de melhorar a qualidade de vida da população. Tem como um dos focos de ação a promoção do envelhecimento saudável ${ }^{11}$. O que remete a necessidade de mais agilidade no sistema de saúde, pois o enveIhecimento traz consigo um expediente reduzido para a procura dos serviços de saúde e deslocamento até os diferentes níveis de atenção. Para os idosos, em especial os mais carentes, simples dificuldades acabam sendo motivo de bloqueio e interrupção da continuidade da assistência à saúde ${ }^{12}$.

Apesar de atribuírem características positivas ao atendimento, alguns participantes do estudo referem-se quanto à demora e pouca disponibilidade de vagas na unidade.

O SUS sofre de um problema crônico ligado a uma inadequada política de pessoal em geral e em particular a falta de profissionais médicos. O fato de manter as portas abertas para quem procura os serviços de atenção básica não deve ser confundido com a garantia de acesso. Apesar de ser, geralmente, caracterizada por obtenção de ações resolutivas para o tipo de problema que interfere na saúde do usuá-
A implantação da ESF facilita o acesso da população aos serviços de saúde, pois trazem uma equipe multiprofissional 
rio. As dificuldades para marcar consultas com especialistas associada a demora na realização de exames acaba fazendo com que haja uma descrença no papel de organização da atenção básica. Já que grande parte da demanda está vinculada ao paradigma biomédico, no qual os usuários buscam sempre por ações curativas e centradas na figura do profissional médico ${ }^{13}$.

Há então a necessidade de que seja feito um planejamento que condiz com as necessidades da população alvo e facilite o acesso da comunidade a unidade ${ }^{14}$.

Associado a isso, a mudança na concepção da população a respeito do paradigma biomédico, necessita do entendimento por parte dos usuários a respeito da importância das diversas clínicasno campo da saúde. Esta mudança deve partir de ações pedagógicas elaboradas pela equipe que evidenciem a eficácia de práticas que confrontem a lógica biomédica ${ }^{13}$.

Nesse sentido, urge promover ações voltadas para as políticas públicas que melhorem a qualidade de vida dos usuários. Ou seja, aconselhar a população a respeito de vícios como tabagismo, estimular atividades físicas, entre diversas outras atividades de educação em saúde. Estas são medidas que podem ser exploradas e trazem resultados positivos quando combinadas. Porém, devemos considerar que a efetividade das orientações individuais e comunitárias se restringe as condições culturais, sociais e existenciais nas diversas regiões do país ${ }^{14}$.

Foi relatado, também, na primeira IC quanto à distribuição de medicamentos e a falta de médicos especialistas na unidade.

A ESF tem sua estrutura desvinculada do modelo médico assistencial centrados na doença. A organização dos serviços da atenção básica por meio da ESF tem como prioridade as ações de promoção, prevenção e recuperação da saúde de forma integral e continuada. Sua expansão pelo território brasileiro se define por um conjunto de ações e serviços que visam muito além da assistência médica, tendo como base a necessidade de reconhecer as necessidades da população através da formação de vínculos entre os usuários e os profissionais. Traz como proposta a família como ponto central, sendo compreendida através de seu ambiente físico e social. Leva os profissionais a terem uma visão ampliada do processo de saúde-doença e das necessidades de intervenções, que não visam somente a prática curativa ${ }^{8}$.

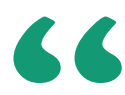

\section{Segundo a Política Nacional de} Atenção Básica (PNAB), a presença de um farmacêutico assim como médicos especialistas, exceto médico generalista ou especialista em saúde da família, não faz parte das especificidades da equipe de Saúde da Família.

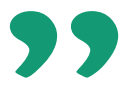

Segundo a Política Nacional de Atenção Básica (PNAB), a presença de um farmacêutico assim como médicos especialistas, exceto médico generalista ou especialista em saúde da família, não faz parte das especificidades da equipe de Saúde da Família. Estes profissionais geralmente fazem parte de equipes atuantes em Núcleos Ampliado de Saúde da Família (NASF). O NASF tem como objetivos ampliar a abrangência e direcionar as ações da Atenção Básica, assim como sua resolubilidade. As equipes atuantes nessas unidades são compostas por profissionais de diferentes áreas do conhecimento, que devem trabalhar de forma integrada complementando os serviços oferecidos pela atenção primária ${ }^{4}$.

Pesquisa realizada com o objetivo de explorar a percepção de usuários e profissionais da ESF sobre o NASF confirma a necessidade de complementação dos serviços prestados pela ESF ao concluir que a incorporação de outros profissionais, através do NASF, fez-se necessária para expandir as ações em saúde e contribuir para uma maior resolutividade na APS. Uma vez que a saúde da família representa uma estratégia prioritária para a reformulação do modelo assistencial, com o intuito de fortalecer a atenção primária no País e garantir a universalidade e a integralidade no cuidado à saúde ${ }^{15}$.

A segunda IC "nenhum" foi extraída dos depoimentos e relataram que nunca conseguiram consulta médica na ESF.

O acolhimento na Atenção Primária é uma das diretrizes principais no que diz respeito à política, ética e estética da Política Nacional de Humanização do SUS no Brasil. É definido como a recepção do cliente nos serviços de saúde. Englobando o ato do profissional de se responsabilizar pelo cliente, ouvindo suas queixas e angústias de forma qualificada, inserindo limites quando preciso, garantindo resolutividade e articulando da melhor forma o usuário a outros serviços de saúde para que seja mantido o cuidado de forma continuada ${ }^{16}$.

Porém, existem obstáculos que impedem o acolhimento de ser realizado da forma como deveria. Alguns dos principais obstáculos são: limitações na estrutura da unidade; questões éticas como ausência de sigilo e privacidade entre o profissional e usuário; postura inadequada, falta de comprometimento do profissional em escutar o cliente que procura por seus serviços na unidade e escassez de profissionais. Os pontos apresentados acabam restringindo o acolhimento por falta de condições básicas de recebimento dos que buscam as unidades. Desrespeitando 
os bens privados destes, juntamente com suas necessidades individuais ${ }^{17}$. A escassez de profissionais também é referida em um estudo realizado com profissionais da Estratégia Saúde da Família, onde apontam como fragilidades importantes além do baixo salário, falta de infraestrutura e carência de insumos de trabalho, o excesso de trabalho por falta de profissionais ${ }^{18}$.

O acolhimento ainda não é completamente sistematizado nos modelos propostos pelo governo na Atenção Básica à Saúde. Podendo ser esta uma das principais justificativas para a insatisfação de usuários dependentes do sistema público de saúde. O acolhimento é uma ferramenta criada para atuar de forma colaborativa com a qualificação dos sistemas de saúde, possibilitando o acesso do usuário a um atendimento realizado de modo justo e integral através de diversos profissionais e setores. Traz consigo a possibilidade do SUS de efetivar seus princípios constitucionais, porém ainda há a necessidade de qualificação na forma com que vem sen- do trabalhado. Pois, se trabalhado de forma desarticulada e pontual pode acabar se tornando uma simples triagem, sendo descaracterizado de sua função principal, que se projeta como a humanização dos serviços de saúde. Desta forma acaba por não alcançar seus propósitos ${ }^{17}$.

\section{CONCLUSÃO}

Os participantes do estudo, apesar de reconhecerem as ações dos profissionais atuantes na ESF e considerarem um grande benefício a proximidade da unidade a moradia, facilitando o acesso, ainda ressaltam a necessidade de melhorias nas condições e serviços ofertados. A implantação dos NASF pode contribuir para um melhor atendimento e satisfação desses usuários.

É perceptível que uma parcela da comunidade está voltada para uma assistência centrada no modelo médico assistencial. Urge a necessidade de conscientização da população quanto a finalidade da ESF, sendo a educação para a saúde uma ferramenta potente para o alcance desse objetivo e o enfermeiro sujeito ativo nesse processo.

Os participantes do estudo que se dizem mais beneficiados, com a ESF, são aqueles que possuem doenças crônicas como a diabetes e hipertensão arterial, já outros participantes relatam dificuldades de acesso. Sendo possível considerar que apesar de exercer importante papel na promoção da saúde e prevenção de agravos, a ESF ainda apresenta falhas na inclusão de toda a comunidade.

O enfermeiro, por exercer um papel fundamental na promoção da saúde, precisa, juntamente com os demais profissionais, criar vínculo com as famílias em sua área de abrangência. Pois a partir da criação de vínculo é possível que o profissional enfermeiro compreenda de uma melhor forma o contexto vivido pelos indivíduos, família e comunidade e diante disso elabore estratégias que condizem com a realidade local.

\section{Referências}

1. Nepomuceno LB, Pontes RJS. O Espaço socioprofissional da Estratégia Saúde da Família sob a perspectiva de psicólogos. Psicol. ciênc. prof. 2017:37(2):289-303.

2. Brasil. Ministério da Saúde. Política Nacional de Atenção Básica [Internet]. Brasília; 2012 [citado 2020 ago. 10]. Disponível em: http://189.28.128.100/ dab/docs/publicacoes/geral/pnab.pdf.

3. Soratto J, Pires DEP, Dornelles $S$, Lorenzetti J. Estratégia Saúde da Família: uma inovação tecnológica em saúde. Texto \& Contexto Enferm. 2015;24(2):584-92

4. Brasil. Ministério da Saúde. Portaria n. 2.436, de 21 de setembro de 2017. Aprova a Política Nacional de Atenção Básica [Internet]. Brasília; 2017 [citado 2020 jul. 19]. Disponivel em: http://bvsms.saude.gov.br/bvs/saudelegis/ gm/2017/ prt2436_22_09_2017.html.

5. Lefêvre F. Discurso do Sujeito Coletivo: nossos modos de pensar; nosso eu coletivo. São Paulo: Andreoli; 2017.

6. Lefèvre F, Lefèvre AMC. Discurso do Sujeito Coletivo: representações sociais e intervenções comunicativas. Texto \& Contexto Enferm. 2014;23(2):5027.

7. Brasil. Ministério da Saúde. Resolução n. 466 de 2012. Trata do respeito pela dignidade humana e pela especial proteção devida aos participantes das pesquisas científicas envolvendo seres humanos [Internet]. Diário Oficial da União; 2013 Jun 13 [citado 2019 fev. 26]. Disponível em: http://conselho.saude.gov.br/resolucoes/2012/Res0466.pdf.

8. Oliveira MAC, Pereira IC. Atributos essenciais da Atenção Primária e a Estratégia Saúde da Família. Rev. bras. enferm. 2013;66(esp):158-64.

9. Jardim LV, Navarro D. Contribuição da ESF no controle de doenças crônicas não transmissíveis. J Health Sci Inst. 2017;35(2):122-6.
10. Tavares GA, Barreto-Filho JAS. Potencial da Estratégia de Saúde da Família no combate às doenças cardiovasculares. Arq. bras. cardiol. 2017:109(6):507-8.

11. Motta LB, Aguiar AC, Caldas CP. Estratégia Saúde da Família e a atenção ao idoso: experiências em três municípios brasileiros. Cad. Saúde Pública. 2011;27(4):779-86.

12. Drumond CH, Borges NL, Machado CH, Horta PM. Transição demográfica e atenção à saúde da pessoa idosa no âmbito da atenção primária à saúde: um estudo de revisão sobre o cenário brasileiro. Rev. APS. 2013;16(3):320-7. 13. Campos RTO, Ferrer AL, Gama CAP, Campos GWS, Trapé TL, Dantas DV. Avaliação da qualidade do acesso na atenção primária de uma grande cidade brasileira na perspectiva dos usuários. Saúde debate. 2014;38(esp):252-64. 14. Norman AH, Tesser CD. Acesso ao cuidado na Estratégia Saúde da Família: equilíbrio entre demanda espontânea e prevenção/promoção da saúde. Saúde Soc. 2015;24(1):165-79.

15. Aciole GG, Oliveira DK. Percepções de usuários e profissionais da saúde da família sobre o Núcleo de Apoio à Saúde da Família. Saúde debate. 2017;41(115):1090-101.

16. Garuzi M, Achitti COM, Sato CA, Rocha AS, Spagnuolo RS. Acolhimento na Estratégia Saúde da Família: revisão integrativa. Rev. panam. salud pública. 2014;35(2):144-9.

17. Coutinho LRP, Barbieri AR, Santos MLM. Acolhimento na Atenção Primária à Saúde: revisão integrativa. Saúde debate. 2015;39(105):514-24. 18. Martins TF, Vieira MV, Souza TMS, Sousa DM, Saldanha KD, Rouberte ESC, et al. Percepção dos profissionais sobre a Estratégia Saúde da Família em um município do interior do Ceará. Rev. bras. promoç. saúde. 2015;28(4):596605 